\title{
DESARROLLO DE COMPETENCIAS SOCIOEMOCIONALES MEDIANTE PLATAFORMA TEC- NOLÓGICA (PROGRAMA EMOTIC): VALORACIÓN DE LOS BENEFICIOS DEL PROGRAMA SEGÚN LOS PARTICIPANTES
}

\author{
Usue de la Barrera $^{1}$, Silvia Postigo-Zegarra ${ }^{2}$, Alicia Tamarit ${ }^{1}$, José-Antonio Gil-Gómez ${ }^{3}$ e Inmacula- \\ da Montoya-Castilla ${ }^{1}$ \\ ${ }^{1}$ Universitat de València, España \\ ${ }^{2}$ Universidad Europea de Valencia, España \\ ${ }^{3}$ Universitat Politècnica de València, España
}

Resumen

Los programas socioemocionales que incorporan las tecnologías en su implementación podrían resultar atractivos en la adolescencia. El objetivo del presente estudio fue analizar la percepción subjetiva de los y las adolescentes sobre sus aprendizajes, el atractivo y las mejoras del programa socioemocional mediante plataforma tecnológica denominado emoTIC. Participaron 84 adolescentes $(M=12,66 ; D T=0,73 ; 41,7 \%$ chicas $)$ que completaron el programa y realizaron la valoración subjetiva. Se analizó la fiabilidad inter-jueces mediante Kappa y Delta. Los resultados mostraron un adecuado acuerdo entre jueces en los aprendizajes $(K=0,85-0,91 ; \Delta=0,91-0,96)$, los aspectos atractivos $(K=$ $0,91-0,96 ; \Delta=0,95-0,96)$ y las mejoras $(K=0,86-0,96 ; \Delta=$ 0,94 - 0,97). Respecto a los aprendizajes, las personas participantes manifestaron haber desarrollado sus competencias emocionales, sus habilidades sociales y su crecimiento personal. Asimismo, los contenidos del programa, el aprendizaje de competencias emocionales y los aspectos técnicos y gráficos les resultaron atractivos. Respecto a las mejoras, sugirieron realizar cambios en la mecánica, el diseño y el funcionamiento de la aplicación. Se propone una versión mejorada denominada emoTIC SPACE. En conclusión, emoTIC podría considerarse una herramienta útil para mejorar las competencias socioemocionales en la adolescencia.

\section{Identificador de ensayos clínicos: NCT04414449}

Palabras clave: Competencias emocionales, Juegos serios, Programas socioemocionales basados en juegos digitales, Diseño mixto, Adolescentes

\section{Abstract}

\begin{abstract}
Social-emotional programmes that incorporate technologies in their implementation could be attractive in adolescence. The aim of this study was to analyse the subjective perception of adolescents about their learning, the appeal and the improvements of the socioemotional programme using a technological platform named emoTIC. Participants were 84 adolescents $(\mathrm{M}=12.66$; $\mathrm{SD}=0.73$; $41.7 \%$ girls), who carried out the subjective assessment. Inter-rater reliability was analysed by Kappa and Delta. The results showed an adequate agreement between raters in learning $(\mathrm{K}=0.85-0.91$; $\Delta=0.91-0.96)$, the appeal $(\mathrm{K}=0.91-0.96 ; \Delta=0.95-0.96)$ and the improvements $(\mathrm{K}=0.86-0.96 ; \Delta=0.94-0.97)$. With regard to learning, adolescents reported that they developed their emotional competences, their social skills and their personal growth. Likewise, the contents of the programme, the learning of emotional competences and the technical and graphic aspects were appealing to them. Regarding the improvements, they suggested making changes in the mechanics, design and operation of the application. An improved version named emoTIC SPACE is proposed. In conclusion, emoTIC could be considered a useful tool to improve social-emotional competences in adolescence.
\end{abstract}

\section{Clinical trial identifier: NCT04414449}

Keywords: Emotional competences, Serious games, socialemotional gam-based programs, Mixed design, Adolescents

Correspondencia.

Inmaculada Montoya-Castilla

inmaculada.montoya@uv.es

Departamento de Personalidad, Evaluación y Tratamientos Psicológicos.

Facultad de Psicología y Logopedia, Universitat de València

Av. Blasco Ibáñez, 21 (46010 Valencia). 
Desarrollo de competencias socioemocionales mediante plataforma tecnológica (Programa emoTIC): valoración de los beneficios del programa según los participantes

\section{Introducción}

\section{Las competencias socioemocionales en la adolescencia}

El modelo de inteligencia emocional de Mayer y Salovey (Mayer y Salovey, 1997) goza de gran apoyo empírico y su fundamentación teórica es sólida (Fernández-Berrocal y Extremera, 2015; Joseph y Newman, 2010; Mayer et al., 2000). Está compuesto por cuatro competencias estructuradas de manera jerárquica (Mayer et al., 2016). La primera competencia es la percepción, la valoración y la expresión emocional. Hace referencia a la capacidad para percibir, identificar y valorar tanto las emociones propias como las de los demás, y expresarlas de forma adecuada. La segunda competencia es la facilitación emocional del pensamiento, entendida como la relación existente entre el pensamiento y la emoción. La tercera competencia es la comprensión de las emociones. Es la habilidad para entender, analizar y predecir las emociones utilizando la información emocional. Implica saber etiquetar y diferenciar las emociones, así como comprender la relación de la emoción con su significado. Por último, la cuarta competencia es la regulación emocional, es decir, la habilidad para utilizar las emociones de forma adaptativa y ser capaz de reducir o disminuir las emociones negativas y aumentar o mantener las positivas, sin reprimirlas o exagerarlas. Desde este enfoque, la inteligencia emocional se conceptualiza como un conjunto de habilidades relacionadas con la percepción, expresión y regulación de las emociones en uno mismo y en los demás, y el uso de esas habilidades para motivar, planificar y lograr objetivos en la vida (Mayer et al., 2004). El desarrollo de las competencias emocionales es un enfoque más general y amplio que el de la inteligencia emocional, que incluye mayor variedad de habilidades emocionales y parece más adecuado para el estudio del desarrollo emocional de niños y adolescentes (Buckley et al., 2003; Saarni, 2000).

Además de las competencias emocionales, existen variables sociales y personales especialmente relevantes en la infancia y adolescencia como son la empatía y la autoestima. Por un lado, la empatía puede ser entendida como una reacción emocional que surge por la percepción de la emoción de otra persona y es congruente con su estado emocional (Garaigordobil y Maganto, 2011). La empatía está relacionada con un mejor manejo de los conflictos (de Wied et al., 2007), habilidad crucial en la relación con los iguales. Por otro lado, la autoestima, desde un enfoque unidimensional, se define como la actitud favorable o desfavorable que tienen las personas hacia sí mismas (Baldwin y Hoffmann, 2002; Rosenberg, 1965; Smith et al., 2014). Durante la adolescencia, las competencias emocionales y la autoestima están relacionadas con mayor bienestar subjetivo y menos problemas emocionales y conductuales (de la Barrera et al., 2019; Escoda y Alegre, 2016; Estevez et al., 2019; Kong et al., 2019). Los estudios sugieren que las y los ado- lescentes que perciben, comprenden y regulan mejor sus emociones, son capaces de afrontar mejor las dificultades emocionales y tienen mayor bienestar (Sánchez-Álvarez et al., 2016). Asimismo, las competencias emocionales parecen influir su funcionamiento social y en las relaciones con los iguales (Elipe et al., 2011; Peachey et al., 2017). Por todo ello, se han desarrollado programas de educación socioemocional en el contexto escolar (e.g., Sarrionandia, 2017; Viguer et al., 2017; Villegas y Raffaelli, 2018) y se ha considerado que su fomento es tan esencial como el académico (Fomina et al., 2020).

\section{Los programas de intervención socioemocionales imple- mentados a través de las tecnologías}

Los programas de intervención para fomentar las competencias emocionales han mostrado su eficacia para desarrollar la inteligencia emocional y, además, han evidenciado su capacidad para incidir de manera indirecta en otras variables tales como el bienestar o la relación con los iguales (p.ej., Hodzic et al., 2018; Schoeps et al., 2018). Estos beneficios parecen ser innegables; por lo que es un reto el desarrollo de programas que, además de ser educativos, sean atractivos en la adolescencia. Actualmente, la juventud está muy familiarizada con el uso de las tecnologías y suele sentir atracción por los juegos, por lo que incluir las tecnologías y gamificar los programas de intervención podría resultar útil para aumentar su motivación por participar (Almeida, 2020; Wouters et al., 2013). En este sentido, los programas de intervención basados en juegos (game-based intervention programs) o los juegos serios (serious games) se están utilizando con el objetivo de potenciar diferentes habilidades (e.g., Grist et al. 2019; Levesque et al., 2017). El aprendizaje basado en juegos digitales (digital gamebased learning) hace referencia a la utilización del poder de entretenimiento de los juegos digitales con fines educativos, intentando lograr un equilibrio entre los elementos de aprendizaje y los elementos de juego (Nussbaum y Beserra, 2014). De la misma manera, los juegos serios son herramientas digitales que facilitan la adquisición de habilidades en un entorno atractivo e interactivo, persiguiendo como fin último un objetivo educativo (Boyle et al., 2016).

A pesar de la existencia de programas de intervención que utilicen las tecnologías para la promoción de la salud mental adolescente (p.ej., Grist et al., 2019), son escasos los que están dirigidos al desarrollo de las competencias socioemocionales. Entre los que existen, por ejemplo, el juego virtual llamado RAGE-control fue diseñado por Kahn et al. (2013) para focalizar la atención de niños y adolescentes hospitalizados en psiquiatría y enseñarles a controlar su activación fisiológica mediante técnicas de relajación. También, Iaosanurak et al. (2016) desarrollaron una intervención para promover el aprendizaje socioemocional a través de las tecnologías y lograron que los niños mejora- 
ran su autocontrol emocional. En España, Cejudo et al. (2019) desarrollaron el videojuego Spock para mejorar la inteligencia emocional en la adolescencia que, además, mejoró el ajuste personal y disminuyó los problemas de conducta.

Los resultados de las investigaciones parecen sugerir que los programas socioemocionales que utilizan las nuevas tecnologías son eficaces para mejorar las competencias emocionales (Cejudo et al., 2019). No obstante, apenas existe evidencia sobre la percepción subjetiva de los propios participantes acerca de los aprendizajes adquiridos. En este sentido, estudios recientes enfatizan la importancia de explorar la experiencia subjetiva de los participantes y la utilidad percibida por los mismos (Postigo-Zegarra, et al., 2019; Twining et al., 2017). Además de la importancia de conocer la percepción subjetiva de las competencias adquiridas, la valoración del programa por parte de los participantes puede ofrecer información acerca de los aspectos que les resultan más atractivos del programa y las posibles mejoras para incorporarlas en una versión posterior.

\section{El presente estudio}

Las investigaciones previas ponen de manifiesto la necesidad de desarrollar programas socioemocionales implementados a través de las tecnologías en los que se analice la valoración de los propios participantes. Por todo ello, el objetivo del presente estudio fue analizar la percepción subjetiva de los y las adolescentes sobre los aprendizajes, el atractivo y las mejoras del programa socioemocional mediante plataforma tecnológica denominado emoTIC. En relación a la valoración de los y las participantes, las hipótesis fueron: (1) valorarán que el programa de intervención ha desarrollado sus competencias socioemocionales (Cejudo et al., 2019); (2) valorarán que ha fomentado la relación con los iguales (Schoeps et al., 2018); y (3) los aspectos atractivos y las mejoras del programa estarán relacionados con el uso de las tecnologías (Wouters et al., 2013).

\section{Método}

\section{Participantes}

Los participantes fueron 84 adolescentes de 11 a 15 años $(M=12,66 ; D T=0,73)$, pertenecientes a los cursos de primero y segundo de Educación Secundaria Obligatoria (E.S.O.) de colegios públicos y concertados de la Comunidad de Madrid. En cuanto al género (femenino, masculino, otro, no contesto), el 41,7\% eran chicas $(n=35)$, el 52,4\% eran chicos $(n=44)$ y el $6,0 \%$ no contestó sobre su género $(n=6)$. El 92,4\% eran adolescentes de nacionalidad española y el 7,6\% provenían de otros países (Rumanía y Venezuela) y llevaban entre 2 y 12 años residiendo en España $(M=7,00 ; D T=4,82)$.
Los criterios de inclusión fueron tres: (1) estar matriculado en primer o segundo curso de la E.S.O. en un centro escolar en España; (2) presentar el consentimiento informado firmado por los padres, madres o tutores legales; y, (3) disponer de un dispositivo móvil como teléfono inteligente o tableta (en su defecto, que el centro pudiera proporcionárselo).

\section{Instrumentos}

Se elaboró una batería de preguntas ad hoc para la recogida de información y se administraron cuestionarios validados en población española adolescente, para evaluar variables relacionadas con las competencias emocionales. Respecto a las variables sociodemográficas, se recogieron datos acerca del género, la edad y el curso escolar. Respecto a la valoración subjetiva del programa, las personas participantes contestaron a tres cuestiones. La primera de ellas versaba sobre los posibles conocimientos adquiridos durante el programa de intervención (¿Qué has aprendido a lo largo de emo$T I C$ ?). La segunda hacía referencia a los aspectos más atractivos del programa (¿Qué es lo que más te ha gustado del programa emoTIC?). Por último, la tercera pregunta se formuló en relación a los posibles cambios o mejoras (¿Qué aspectos se podrían mejorar del programa emoTIC?). En el presente artículo se muestran los resultados de esta valoración subjetiva de los participantes.

\section{Estructura del programa de intervención}

El programa de intervención emoTIC está insertado en una plataforma tecnológica, similar a un serious game, cuyo objetivo general es el desarrollo y la promoción de las competencias socioemocionales en la adolescencia. Este programa se basa en el Modelo de Habilidad de Inteligencia Emocional de Mayer y Salovey (Mayer y Salovey, 1997; Mayer et al., 2016). El programa constaba de cuatro sesiones grupales en el aula y doce actividades individuales a realizar en casa, todas ellas guiadas mediante el dispositivo móvil (Tabla 1).

Todas las sesiones en grupo siguieron una misma estructura. En primer lugar, cada adolescente realizaba una autoexploración emocional para detectar cómo se sentía. Seguidamente, se realizaba una dinámica de grupo según el tema de la sesión, un debate y una reflexión grupal. Finalmente, el docente realizaba una recapitulación y un resumen de la sesión. En cuanto a las actividades individuales para casa, cada adolescente completaba tres cada semana para reforzar lo trabajado en clase. Las tres actividades semanales estaban relacionadas con el área del Modelo de Inteligencia Emocional de Mayer y Salovey que se había trabajado en clase. La estructura era similar en los cuatro bloques. La primera actividad se dedicaba a repasar la adquisición de conceptos básicos. La segunda se centraba en la autoexploración, el autoconocimiento y la auto-reflexión. Por 
Desarrollo de competencias socioemocionales mediante plataforma tecnológica (Programa emoTIC): valoración de los beneficios del programa según los participantes

último, la tercera ponía a prueba las habilidades emocionales relativas a la temática trabajada en la sesión de clase correspondiente.

Para aumentar el atractivo y la motivación del alumnado, el programa fue diseñado como una aventura espacial y el objetivo que se les planteaba a era reconstruir una nave espacial para poder regresar a la Tierra. Las piezas de la nave espacial se conseguían superando las sesiones en el aula y, como refuerzo, se les ofrecían puntos por superar las actividades de casa, que podían utilizar para acceder a varios juegos (diseñados para esta plataforma y con temática también espacial). La aplicación podía ser utilizada en teléfonos inteligentes (smartphones) o en tabletas (tablets), y estaba disponible para iOS y Android.

Tabla 1.

Sesiones, actividades, objetivos y contenidos del programa de intervención

\begin{tabular}{lll}
\hline Sesiones grupales & Objetivos & Contenidos \\
Actividades individuales &
\end{tabular}

\begin{tabular}{ll}
\hline I. ¿Qué son las emociones? & Favorecer las cohesión de grupo y desa- \\
1. ¿Quién la tiene? & rrollar la percepción, la expresión y la \\
2. DiMOOD I & valoración emocional \\
3. Caras ocultas &
\end{tabular}

3. Caras ocultas

II. Emociones y pensamientos

1. Optimismo inteligente

2. DiMOOD II

3. ¿Qué está pasando?

III. Entendiendo mis emociones

1. Focusing emocional

Comprender las emociones y desarrollar la autoestima

Entender la relación que existe entre las emociones y los pensamientos

\section{Red de emociones}

3. Tus fortalezas

IV. ¿Cómo puedo manejar mis emociones?

Potenciar la regulación emocional
1. El semáforo
2. Estrategias emocionales
3. Tienes un mensaje

Conceptos básicos relacionados con las emociones.

- Identificación y valoración de emociones propias y de los demás.

- $\quad$ Expresión de las propias emociones.

- $\quad$ Facilitación emocional del pensamiento.

- $\quad$ Experimentación de la influencia de las emociones en el pensamiento $\mathrm{y}$ viceversa.

- $\quad$ Toma de perspectiva.

Comprensión de las funciones de las emociones.

- $\quad$ Etiquetado de las emociones y comprensión de las emociones complejas y mixtas.

- $\quad$ Fomento de la autoestima.

- $\quad$ Conocimiento y práctica de las estrategias de regulación emocional.

- $\quad$ Regulación emocional para la promoción del crecimiento emocional e intelectual.

- Resolución de situaciones emocionales conflictivas. 


\section{Procedimiento}

En primer lugar, el investigador principal del proyecto contactó con los equipos directivos de los centros escolares y les expuso los principales objetivos de la investigación. Seguidamente, el equipo de dirección de los centros interesados en participar, organizó una reunión con las madres y los padres para presentarles el estudio. Los padres y las madres, así como los adolescentes participantes firmaron el consentimiento informado. El profesorado realizó un curso de formación para aprender a utilizar emoTIC en las aulas y llevó a cabo la implementación del programa en los centros escolares durante cuatro semanas en el horario de tutoría. Para el desarrollo del programa fue necesaria la utilización de teléfonos inteligentes y tabletas. Este estudio fue aprobado por el Comité Ético de la Universitat de València (H152865096049), y para la obtención de datos se siguieron las directrices marcadas por la Declaración de Helsinki (World Medical Association, 2013).

\section{Análisis de datos}

El estudio siguió un diseño mixto que integró datos cualitativos y cuantitativos. Para el análisis de los datos, se conformó un listado de categorías que engloban cada uno de los temas o ideas propuestos por los y las adolescentes. Siguiendo las directrices de Denzin y Lincoln (2008), dos jueces de manera independiente realizaron la asignación de cada uno de estos temas o ideas a una subcategoría. Después, estas subcategorías se agruparon en categorías básicas, para las que se calcularon los índices de acuerdo interjueces Kappa $(\mathrm{K})$ y Delta $(\Delta)$. Una vez calculados, se analizaron conjuntamente aquellos ítems en los que no hubo acuerdo y se realizó un debate entre ambos jueces hasta alcanzar un $100 \%$ de acuerdo en el sistema de categorías y la inclusión de cada uno de los ítems. Finalmente, se calcularon las frecuencias y los porcentajes de cada categoría y subcategoría.

\section{Resultados}

\section{Análisis de los aprendizajes}

Los resultados sobre el aprendizaje de los y las adolescentes se clasificaron en tres categorías básicas: (1) competencias emocionales; (2) habilidades sociales; y (3) desarrollo personal. Se registró un total de 187 referencias repartidas entre las tres categorías y fueron las competencias emocionales las que obtuvieron mayor número de referencias (Tabla 2).

Tabla 2.

Sistema categorial de aprendizajes percibidos del programa

\begin{tabular}{|c|c|c|c|c|}
\hline \multirow{2}{*}{ Categorías básicas y subcategorías } & \multicolumn{2}{|c|}{ Frecuencias } & \multicolumn{2}{|c|}{ Acuerdo inter-jueces } \\
\hline & $n$ & $\%$ & $K(S E)$ & $\Delta(S E)$ \\
\hline Competencias emocionales & 138 & 73,80 & $0,91(0,03)$ & $0,93(0,03)$ \\
\hline Percepción, valoración y expresión emocional & 58 & 31,02 & & \\
\hline Facilitación emocional del pensamiento & 14 & 7,49 & & \\
\hline Comprensión emocional & 22 & 11,76 & & \\
\hline Regulación emocional & 44 & 23,53 & & \\
\hline Habilidades sociales & 28 & 14,97 & $0,85(0,05)$ & $0,91(0,03)$ \\
\hline Cohesión de grupo y compañerismo & 19 & 10,16 & & \\
\hline Empatía & 9 & 4,81 & & \\
\hline Desarrollo personal & 21 & 11,23 & $0,89(0,05)$ & $0,96(0,02)$ \\
\hline Autoestima & 7 & 3,74 & & \\
\hline Valores y respeto & 14 & 7,49 & & \\
\hline TOTAL & 187 & 100,00 & & \\
\hline
\end{tabular}

Nota. $n=$ número de referencias. $\%=$ porcentaje de referencias. $\mathrm{K}=$ Kappa, índice de acuerdo inter-jueces. $\Delta=$ Delta, índice de acuerdo inter-jueces; $S E=$ error estándar. 
Desarrollo de competencias socioemocionales mediante plataforma tecnológica (Programa emoTIC): valoración de los beneficios del programa según los participantes

\section{(1) Competencias emocionales}

La primera categoría hace referencia a las competencias emocionales, que corresponden a las cuatro competencias propuestas desde el modelo de habilidad de inteligencia emocional de Mayer y Salovey (Mayer y Salovey, 1997; Mayer et al., 2016). Tras la implementación de emoTIC, afirmaron haber mejorado en las cuatro competencias emocionales, especialmente en percepción, valoración y expresión, y en regulación emocional.

Percepción, valoración y expresión emocional. Las personas participantes indicaron que el programa aumentó su capacidad para percibir y detectar las emociones. Algunas de sus respuestas sobre lo aprendido fueron: "A conocer mis emociones", "A detectar las emociones", "A reconocer mis emociones", "A detectar emociones de los demás". Asimismo, se percataron de la importancia de valorar las emociones y desarrollaron su propia capacidad, aspectos que quedan reflejados en afirmaciones como: "A valorar más mis sentimientos", "Que los sentimientos son muy importantes para la vida", "Que hay que hacer más caso a las emociones", "A saber valorar mis emociones". Además, el programa pareció reforzar sus habilidades de expresión de las emociones, aspectos evidenciados en respuestas como: "A expresar mis sentimientos", "A decir mis sentimientos", "A expresarme".

Facilitación emocional del pensamiento. Los y las adolescentes manifestaron haber aprendido la relación entre las emociones y los pensamientos, así como a ser conscientes de que se afectan mutuamente. Por ejemplo: "Pensar en mis emociones", "Que las emociones tienen a veces un impacto grande en nosotros", "Reconocer mis emociones y pensar en ellas".

Comprensión emocional. Desarrollaron su comprensión de las emociones en un sentido amplio, así como ciertos aspectos específicos de la comprensión emocional, como son: la capacidad para distinguir entre emociones y para conocer su función. Algunos ejemplos de estos aprendizajes son los siguientes: "A entender las emociones", "A comprender las emociones", "Los diversos estados emocionales", "A saber para qué sirven las emociones", "Los tipos de emociones".

Regulación emocional. Manifestaron haber aprendido acerca del manejo de las emociones con afirmaciones tales como: "A saber cómo controlar mis emociones", "A regular las emociones", "Que tengo que controlar mis impulsos", "A controlar mis sentimientos", "Aprender a controlar las emociones". Asimismo, indicaron haber adquirido algunas técnicas de regulación emocional trabajadas en el programa para reducir, mantener o aumentar la intensidad de las emociones, así como para fomentar emociones agradables, como muestran los siguientes ejemplos: "No dar tantas vueltas a las cosas", "A intentar sentirme mejor", "Respirar", "A no enfadarme y estar de buen humor", "Formas de escuchar y relajarme con los ojos cerrados", "Técnicas de relajación”.

\section{(2) Habilidades sociales}

La segunda categoría hace referencia a las habilidades sociales y las relacionadas con la interacción interpersonal. Los y las adolescentes indicaron haber mejorado, especialmente, su cohesión como grupo y su empatía.

Cohesión de grupo y compañerismo. Las y los adolescen- tes consideran a los iguales personas de referencia (OlivaDelgado, 2011), por lo que el clima de aula, la cooperación y el compañerismo adquieren especial relevancia durante este período del ciclo vital. Tras la implementación de emo$T I C$, manifestaron tener una mejor cohesión de grupo a través de afirmaciones como las siguientes: "He aprendido a ayudar a los demás", "Apoyar a quien está mal", "Que tenemos que valorar más a los amigos", "A agradecer a los demás", "A ayudar a mis amigos", "A confiar en los otros", "Socializar", "He aprendido a querer a la gente", "A tratar bien a mis compañeros", "No pelear y no discutir", "Valorar a los demás".

Empatía. Indicaron que el programa de intervención desarrolló su capacidad empática. Algunos ejemplos de las personas participantes fueron: "A sentir los sentimientos de los demás", "A empatizar”, "A notar las emociones de los demás", "A tener empatía con los demás niños".

\section{(3) Desarrollo personal}

La tercera categoría hace referencia al desarrollo personal los y las adolescentes que realizaron el programa emoTIC, centrándose especialmente en la autoestima y los valores.

Autoestima. Las personas participantes refieren haber mejorado su autoestima, entendiéndola como la actitud positiva que tienen hacia sí mismas. El fomento de la autoestima se reflejó en afirmaciones como: "He aprendido a ser bueno conmigo mismo", "He aprendido a valorarme más", "He aprendido a creer en mí misma", "A confiar más en mí misma".

Valores y respeto. Manifestaron la adquisición de valores durante la implementación del programa emoTIC. Algunas de las afirmaciones realizadas en relación a esta subcategoría fueron: "Paciencia", "Respetar", "Valorar", "Tenemos que esforzarnos por conseguir las cosas".

\section{Análisis de los aspectos atractivos}

Lo atractivo e interesante que resulte el programa favorecerá que las personas se impliquen y que realicen las actividades, de forma que puedan beneficiarse de los aprendizajes en competencias emocionales. Los resultados sobre aspectos del programa considerados por las y los adolescentes como más atractivos se clasificaron en cuatro categorías básicas: (1) contenido del programa; (2) aprendizajes; (3) aspectos técnicos y gráficos; y (4) aspectos generales y lúdicos. Se registró un total de 134 referencias repartidas entre las categorías y fue el contenido del programa la categoría que obtuvo más referencias (Tabla 3).

\section{(1) Contenido del programa}

Dentro del contenido del programa, se observaron tres subcategorías referidas a las misiones, los entrenamientos y los juegos.

Las misiones. Las misiones eran las sesiones que realizaban de forma grupal en la hora de clase. Por ejemplo, afirmaron: "Lo que más me ha gustado son las misiones", "Me ha gustado el ejercicio del teatro", "Me ha gustado la misión 2", "Me ha gustado la relajación".

Los entrenamientos. Los entrenamientos eran las actividades individuales que realizaban en casa guiados por la aplicación. Algunas afirmaciones fueron: "Me han gustado los entrenamientos", "El entrenamiento de las caras ocultas", "Me ha gustado el entrenamiento de los mensajes".

Los juegos. Eran los mini-juegos que podían adquirir a 
través de los puntos que obtenían como recompensa por la realización de las actividades individuales. En este sentido, manifestaron que les habían gustado a través de afirmaciones como: "Los juegos", "Me han gustado los minijuegos".

\section{(2) Aprendizajes}

Uno de los aspectos que más les ha gustado del programa según su propia percepción subjetiva son los aprendizajes obtenidos. Esta categoría engloba dos subcategorías: las competencias emocionales y la utilidad.

Las competencias emocionales. Por un lado, afirmaron que lo que más les ha gustado es aprender sobre las competencias emocionales, por ejemplo: "Me ha gustado decir mis sentimientos", "Me ha gustado saber cómo controlar mis emociones", "Me ha gustado saber cómo me siento".

Utilidad. Por otro lado, lo que más les ha gustado es la utilidad que atribuyen al aprendizaje sobre las emociones; algunos ejemplos son los siguientes: "Me ha gustado cómo me ha hecho reflexionar sobre la amistad y las actitudes", "Me ha gustado que me han enseñado cosas sobre los sentimientos", "Me ha ayudado a aprender más", "Me ha gustado entender el rol que tienen las emociones para aprender".

\section{(3) Aspectos técnicos y gráficos}

Al tratarse de una aplicación para dispositivos móviles, como teléfonos inteligentes o tabletas, la parte técnica y gráfica toma especial relevancia. Se distinguieron dos subcategorías: la mecánica de juego y el diseño.

Mecánica de juego. Algunas personas manifestaron que les había gustado la mecánica del juego con afirmaciones como: "Me ha gustado que sea un juego", "Me ha gustado la facilidad", "El uso de los puntos".

Diseño. Otras personas participantes destacaron que lo que más les había gustado eran aspectos del diseño de emoTIC, por ejemplo: "Me han gustado los avatares", "La nave", "La cara del alienígena", "El sonido de la campanita", "Lo que más me ha gustado son los gráficos".

\section{(4) Aspectos generales y lúdicos}

Hubo adolescentes que valoraron el programa emoTIC en términos generales, informando que les había gustado "todo" de la aplicación. Asimismo, otros manifestaron que les había resultado divertido, especialmente por el uso de la tecnología y la posibilidad de compartir. Por ejemplo: "Ha sido divertido", "Me ha gustado compartir y divertirme", "Me ha gustado estar con la Tablet".

\section{Análisis de las mejoras}

Además de los aprendizajes y los puntos atractivos del programa, se exploraron las posibles mejoras según la percepción de los y las adolescentes participantes. Los resultados se clasificaron en tres categorías básicas: (1) contenido del programa; (2) aspectos técnicos y gráficos; y (3) aspectos generales. Se registró un total de 78 referencias repartidas entre las categorías y fueron los aspectos técnicos y gráficos los que tenían más referencias (Tabla 4).

Tabla 3.

Sistema categorial de los aspectos atractivos percibidos del programa

\begin{tabular}{|c|c|c|c|c|}
\hline \multirow{2}{*}{ Categorías básicas y subcategorías } & \multicolumn{2}{|c|}{ Frecuencias } & \multicolumn{2}{|c|}{ Acuerdo inter-jueces } \\
\hline & $n$ & $\%$ & $K(S E)$ & $\Delta(S E)$ \\
\hline Contenido del programa & 72 & 53,73 & $0,96(0,02)$ & $0,96(0,02)$ \\
\hline Misiones & 35 & 26,12 & & \\
\hline Entrenamientos & 22 & 16,42 & & \\
\hline Juegos & 15 & 11,19 & & \\
\hline Aprendizajes & 29 & 21,64 & $0,93(0,04)$ & $0,95(0,03)$ \\
\hline Competencias emocionales & 21 & 15,67 & & \\
\hline Utilidad & 8 & 5,97 & & \\
\hline Aspectos técnicos y gráficos & 19 & 14,18 & $0,91(0,05)$ & $0,95(0,02)$ \\
\hline Mecánica & 7 & 5,22 & & \\
\hline Diseño & 12 & 8,96 & & \\
\hline Aspectos generals y lúdicos & 14 & 10,45 & $0,94(0,04)$ & $0,96(0,02)$ \\
\hline Diversión & 9 & 6,72 & & \\
\hline Todo & 5 & 3,73 & & \\
\hline TOTAL & 134 & 100,00 & & \\
\hline
\end{tabular}

Nota. $n=$ número de referencias. $\%=$ porcentaje de referencias. $\mathrm{K}=$ Kappa, índice de acuerdo inter-jueces. $\Delta=$ Delta, índice de acuerdo inter-jueces; $S E=$ error estándar. 
Desarrollo de competencias socioemocionales mediante plataforma tecnológica (Programa emoTIC): valoración de los beneficios del programa según los participantes

Tabla 4.

Sistema categorial de los aspectos a mejorar del programa

\begin{tabular}{lcccc}
\hline \multirow{2}{*}{ Categorías básicas y subcategorías } & \multicolumn{3}{c}{ Frecuencias } & \multicolumn{2}{c}{ Acuerdo inter-jueces } \\
\cline { 2 - 5 } & $\boldsymbol{n}$ & $\boldsymbol{\%}$ & $\boldsymbol{K}(\boldsymbol{S E})$ & $\boldsymbol{\Delta}(\boldsymbol{S E})$ \\
\hline Contenido del programa & 16 & 20,52 & $0,86(0,07)$ & $0,94(0,03)$ \\
$\quad$ Misiones y entrenamientos & 13 & 16,67 & & \\
Juegos & 3 & 3,85 & & \\
Aspectos técnicos y gráficos & 43 & 55,12 & $0,94(0,03)$ & $0,95(0,03)$ \\
Mecánica & 12 & 15,38 & & \\
Diseño & 16 & 20,51 & & \\
Funcionamiento & 15 & 19,23 & & \\
Aspectos generales & 19 & 24,36 & $0,97(0,02)$ \\
Nada & 18 & 23,08 & & \\
Todo & 1 & 1,28 & & \\
TOTAL & 78 & 100,00 & & \\
\hline
\end{tabular}

Nota. $n=$ número de referencias. $\%=$ porcentaje de referencias. $\mathrm{K}=$ Kappa, índice de acuerdo inter-jueces. $\Delta=$ Delta, índice de acuerdo inter-jueces; $S E=$ error estándar. 


\section{(1) Contenido del programa}

Las y los adolescentes informaron que el programa se podía mejorar respecto a su contenido, especialmente atendiendo a las misiones y entrenamientos y a los juegos.

Misiones y entrenamientos. Sobre las misiones y entrenamientos, proponen cambios sobre la duración, la cantidad, el contenido y el tipo; por ejemplo: "Que sean más largos", "Más misiones", "Que haya más actividades de relajación o paz", "Que haya otro tipo de misiones", "Hacer las preguntas de otra manera", "Que hubiese algo más aparte de emociones y sentimientos".

Juegos. De forma casi unánime, sugieren la inclusión de más minijuegos, es decir, los juegos que se adquieren a través de los puntos obtenidos tras la realización de las actividades para casa. Algunos ejemplos fueron: "Poner más juegos", "Que tenga más juegos”, "Más juegos”.

\section{(2) Aspectos técnicos y gráficos}

Los y las adolescentes han propuesto cambios en la mecánica, el diseño y el funcionamiento de la aplicación de emoTIC.

Mecánica. Las personas participantes cambiarían el uso de los puntos y la estructura, por ejemplo: "Cambiaría los puntos", "Pondría que haya más puntos", "Que no sea siempre igual".

Diseño. Proponen mejoras en los gráficos, los avatares y los sonidos; por ejemplo: "Cambiaría los gráficos", "Cambiaría los personajes", "La ropa del avatar", "Me gustaría que le pusieran música”, "Más avatares”.

Funcionamiento. Sugieren cambios para mejorar el funcionamiento de la aplicación y señalan algunos errores técnicos; por ejemplo: "Que no tarden tanto los audios", "Que se pueda guardar la partida", "El funcionamiento", "Que no hubiera que poner tantos códigos".

\section{(3) Aspectos generales}

Los y las adolescentes también realizaron valoraciones generales del programa. Se obtuvo una referencia en la que un adolescente cambiaría todo de la aplicación ("Todo") y 18 referencias en las que se comenta que no cambiarían nada ("Nada", "No cambiaría nada", "Nada, está genial, a mi me ha encantado").

\section{Discusión}

El propósito del presente estudio fue valorar la percepción subjetiva de los y las adolescentes sobre el programa socioemocional, mediante plataforma tecnológica, denominado emoTIC.

La primera hipótesis planteaba que las personas participantes valorarían que el programa de intervención desarrollaría sus competencias socioemocionales. Tras el análisis de las valoraciones subjetivas sobre los aprendizajes adquiridos, los resultados sugieren que las personas participantes perciben que emoTIC desarrolla y fomenta las cuatro competencias emocionales del modelo de Mayer y Salovey (Mayer et al., 2016). Los y las adolescentes parecen haber progresado especialmente en su capacidad para percibir, valorar y expresar sus emociones, así como en la habilidad para manejar y regular sus sentimientos y sus estados emocionales. Asimismo, también manifiestan haber aprendido a entender sus emociones y a conocer la relación que existe entre el pensamiento y las emociones. Estos hallazgos van en la línea de investigaciones previas que sugieren que las competencias emocionales son susceptibles de ser potenciadas, especialmente durante la adolescencia (Cejudo et al., 2019).

En la segunda hipótesis, se planteaba que el programa de intervención fomentaría la relación con los iguales. En este sentido, los resultados obtenidos parecen ir en la línea de investigaciones previas y sugieren que los programas de intervención socioemocionales pueden mejorar la relación con los pares (Schoeps et al., 2018), al menos desde el punto de vista de las propias personas participantes. Durante esta etapa del ciclo vital, los iguales toman especial relevancia (Oliva-Delgado, 2011) y pueden surgir dificultades en la relación con los compañeros y las compañeras. La empatía está relacionada con un mejor manejo de esos conflictos (de Wied et al., 2007) y los resultados del presente estudio sugieren que los y las adolescentes consideran que han mejorado el compañerismo y la empatía tras el uso de emoTIC. El programa comprendía sesiones en grupo que se realizaban en el aula y permitían al estudiantado conocer mejor a sus pares, expresar sus propias emociones y compartir situaciones personales con sus iguales. De esta manera, las y los adolescentes refieren que han sido capaces de sentir con los otros y de mejorar la cohesión como grupo. Asimismo, cabe destacar que además de las competencias emocionales y las habilidades sociales, manifestaron haber mejorado su autoestima, actitud relacionada con altos niveles de bienestar subjetivo y bajos niveles de problemas emocionales y conductuales (de la Barrera et al., 2019; Escoda y Alegre, 2016).

La tercera hipótesis hacía referencia al atractivo del programa y las posibles mejoras. Respecto al atractivo del programa, se planteó que estaría relacionado con el uso de las tecnologías. Los estudios sugieren que la gamificación y el uso de tecnologías podrían resultar atractivos para los adolescentes (Wouters et al., 2013) y los resultados de este estudio parecen ir parcialmente en la línea. En este sentido, las personas participantes indicaron haberse divertido utilizando emoTIC y les gustaron aspectos relacionados con la mecánica del juego y la estética de la aplicación. Sin embargo, los aspectos más atractivos para están referidos a los 
Desarrollo de competencias socioemocionales mediante plataforma tecnológica (Programa emoTIC): valoración de los beneficios del programa según los participantes

contenidos tratados y a los aprendizajes obtenidos. El alumnado manifestó el atractivo de las misiones (sesiones grupales realizadas en clase) y los entrenamientos (actividades individuales realizadas en casa), así como de juegos proporcionados como refuerzo tras la realización de las actividades. Asimismo, consideraron que la incorporación de las competencias emocionales les había resultado atractiva y útil para su vida. En este sentido, las valoraciones de los y las adolescentes parecen reforzar la importancia del fomento de las competencias socioemocionales en el contexto escolar (Fomina et al., 2020; Villegas y Raffae1li, 2018). Respecto a las posibles mejoras del programa, los resultados van en la línea de la hipótesis planteada y sugieren que podría mejorarse la mecánica, el diseño y el funcionamiento del programa. Proponen mejorar la mecánica de juego incorporando un mejor sistema de distribución de puntos y evitando que sea repetitivo. Respecto al diseño, sugieren mejorar los gráficos, incluir música, añadir avatares, cambiar algunos personajes e incorporar otro tipo de ropa; es decir, proponen que el juego sea más inmersivo y lúdico. En relación al funcionamiento, recomiendan la eliminación de códigos para el avance del juego, lograr que los audios de la aplicación funcionen bien y que se pueda guardar la partida. Esto es, cuestiones técnicas para mejorar el funcionamiento de la propia aplicación.

Las valoraciones proporcionadas por los y las adolescentes participantes en relación a emoTIC han sido utilizadas para desarrollar una nueva versión, emoTIC SPACE. Principalmente, se han realizado cambios en dos bloques: (1) aspectos técnicos y (2) contenidos. En relación al primer bloque, se han corregido los errores técnicos, se ha mejorado el diseño gráfico del juego y se ha transformado en una herramienta autoaplicable accesible desde cualquier dispositivo tecnológico (p.ej., ordenadores, teléfonos inteligentes, pizarras electrónicas o tabletas). En relación al segundo bloque, se han incorporado más áreas de trabajo, por ejemplo, la toma de perspectiva, el aprecio a la diversidad, el manejo del estrés, la percepción de autoeficacia y la asertividad; y se proporciona más feedback en todas las actividades que realizan los participantes. Por tanto, la nueva versión de emoTIC es un serious game interactivo, con diversos retos y actividades con un diseño gráfico atractivo que, además, proporciona información de acuerdo al desempeño. Se ha diseñado de forma que sea lo más inmersivo posible para lograr una mayor implicación y motivación. Así, se realizan todas las actividades en el marco de un entorno ficticio que se desarrolla en cuatro Territorios, cada uno con un paisaje distinto, en el planeta E-MOOD. Un hilo argumental acompaña a través de junglas sombrías y montañas nevadas que harán que se sumerja en la historia y experimenten este programa como si fuera un estimulante videojuego. Además, el propio emoTIC SPACE tiene incorporado un sistema de refuerzo, premiando sus participantes cuando completan un reto correctamente.

Este estudio no está exento de limitaciones. Primero, únicamente se contempla la percepción subjetiva que realizan las personas participantes sobre sus aprendizajes. Sería necesario utilizar otro tipo de medidas de forma conjunta, como los autoinformes o las tareas de ejecución, que determinen la mejora en el desarrollo de las competencias, además de las propias valoraciones. En segundo lugar, sería necesario realizar una valoración del programa a largo plazo para analizar si los aprendizajes adquiridos se han integrado y permanecen en el tiempo. A pesar de las limitaciones, este estudio presenta tres fortalezas. En primer lugar, se ofrece un programa de educación socioemocional para adolescentes que incluye la tecnología para su implementación, aumentando así su atractivo. En segundo lugar, se incorpora la percepción de las personas participantes acerca de los aprendizajes obtenidos. Finalmente, se incluye un análisis de los aspectos más atractivos del programa y de las posibles mejoras que han sido incluidas en una nueva versión de emoTIC.

En conclusión, el programa de educación socioemocional mediante plataforma tecnológica llamado emoTIC se muestra útil para mejorar las competencias emocionales, las habilidades sociales y el crecimiento personal en la adolescencia. Al incorporar la tecnología en su implementación, aumenta el atractivo que tiene en esta etapa evolutiva y mejora su compromiso con el programa. Por todo ello, se considera un programa idóneo para ser llevado a cabo en el contexto educativo.

\section{Conclusión}

Para finalizar, resaltar una serie de conclusiones sobre la utilidad del programa emoTIC que se derivan del presente estudio. En primer lugar, se ofrece un programa para el desarrollo de las competencias socioemocionales que se realiza sobre una plataforma tecnológica. La inclusión de la tecnología y la gamificación en el área de las competencias socioemocionales conlleva un gran avance para la intervención y la promoción de la salud. En este sentido, permiten hacer más atractivos los programas de educación emocional en la adolescencia y aumentan su adherencia (Almeida, 2020; Iaosanurak et al., 2016). El presente estudio contribuye a evidenciar el impacto positivo que tienen las tecnologías para el desarrollo de las competencias socioemocionales en la adolescencia. En segundo lugar, se han analizado las valoraciones subjetivas de los y las adolescentes en relación al programa emoTIC, lo que permite explorar los aprendizajes adquiridos desde su propia perspectiva. Tras el estudio, se aporta mayor evidencia sobre la utilidad de la información que nos proporcionan las personas que participan, dado que permiten enriquecer los programas socioemocionales y guiar los cambios que se han de realizar para 
mejorarlos (Postigo-Zegarra, et al., 2019; Twining et al., 2017). Finalmente, el programa emoTIC atiende la necesidad del desarrollo socioemocional de los y las adolescentes, aspecto que se ha considerado tan esencial como el académico en el contexto escolar (Fomina et al., 2020). Las competencias emocionales, las habilidades sociales y el crecimiento personal son factores protectores de la salud mental adolescente y su desarrollo mediante programas atractivos implementados a través de las tecnologías pueden prevenir la aparición de problemas psicológicos en la adolescencia. Tras este estudio, se ofrece un programa socioemocional que permite promover la salud mental adolescente y favorecer las relaciones con los iguales en el contexto escolar. De esta manera, las implicaciones del programa van más allá del alumnado y pueden repercutir positivamente en otros agentes del contexto escolar, como son el profesorado, y en el seno familiar de los y las adolescentes.

\section{(1) Contenido del programa}

Las y los adolescentes informaron que el programa se podía mejorar respecto a su contenido, especialmente atendiendo a las misiones y entrenamientos y a los juegos.

Misiones y entrenamientos. Sobre las misiones y entrenamientos, proponen cambios sobre la duración, la cantidad, el contenido y el tipo; por ejemplo: "Que sean más largos", "Más misiones", "Que haya más actividades de relajación o paz", "Que haya otro tipo de misiones", "Hacer las preguntas de otra manera", "Que hubiese algo más aparte de emociones y sentimientos".

Juegos. De forma casi unánime, sugieren la inclusión de más minijuegos, es decir, los juegos que se adquieren a través de los puntos obtenidos tras la realización de las actividades para casa. Algunos ejemplos fueron: "Poner más juegos", “Que tenga más juegos”, "Más juegos”.

\section{(2) Aspectos técnicos y gráficos}

Los y las adolescentes han propuesto cambios en la mecánica, el diseño y el funcionamiento de la aplicación de emoTIC.

Mecánica. Las personas participantes cambiarían el uso de los puntos y la estructura, por ejemplo: "Cambiaría los puntos", "Pondría que haya más puntos", "Que no sea siempre igual".

Diseño. Proponen mejoras en los gráficos, los avatares y los sonidos; por ejemplo: "Cambiaría los gráficos", "Cambiaría los personajes", "La ropa del avatar”, "Me gustaría que le pusieran música”, "Más avatares”.

Funcionamiento. Sugieren cambios para mejorar el funcionamiento de la aplicación y señalan algunos errores técnicos; por ejemplo: "Que no tarden tanto los audios", "Que se pueda guardar la partida", "El funcionamiento", "Que no hubiera que poner tantos códigos".

\section{(3) Aspectos generales}

Los y las adolescentes también realizaron valoraciones generales del programa. Se obtuvo una referencia en la que un adolescente cambiaría todo de la aplicación ("Todo") y 18 referencias en las que se comenta que no cambiarían nada ("Nada", "No cambiaría nada", "Nada, está genial, a mi me ha encantado").

\section{Discusión}

El propósito del presente estudio fue valorar la percepción subjetiva de los y las adolescentes sobre el programa socioemocional, mediante plataforma tecnológica, denominado emoTIC.

La primera hipótesis planteaba que las personas participantes valorarían que el programa de intervención desarrollaría sus competencias socioemocionales. Tras el análisis de las valoraciones subjetivas sobre los aprendizajes adquiridos, los resultados sugieren que las personas participantes perciben que emoTIC desarrolla y fomenta las cuatro competencias emocionales del modelo de Mayer y Salovey (Mayer et al., 2016). Los y las adolescentes parecen haber progresado especialmente en su capacidad para percibir, valorar y expresar sus emociones, así como en la habilidad para manejar y regular sus sentimientos y sus estados emocionales. Asimismo, también manifiestan haber aprendido a entender sus emociones y a conocer la relación que existe entre el pensamiento y las emociones. Estos hallazgos van en la línea de investigaciones previas que sugieren que las competencias emocionales son susceptibles de ser potenciadas, especialmente durante la adolescencia (Cejudo et al., 2019).

En la segunda hipótesis, se planteaba que el programa de intervención fomentaría la relación con los iguales. En este sentido, los resultados obtenidos parecen ir en la línea de investigaciones previas y sugieren que los programas de intervención socioemocionales pueden mejorar la relación con los pares (Schoeps et al., 2018), al menos desde el punto de vista de las propias personas participantes. Durante esta etapa del ciclo vital, los iguales toman especial relevancia (Oliva-Delgado, 2011) y pueden surgir dificultades en la relación con los compañeros y las compañeras. La empatía está relacionada con un mejor manejo de esos conflictos (de Wied et al., 2007) y los resultados del presente estudio sugieren que los y las adolescentes consideran que han mejorado el compañerismo y la empatía tras el uso de emoTIC. El programa comprendía sesiones en grupo que se realizaban en el aula y permitían al estudiantado conocer mejor a sus pares, expresar sus propias emociones y compartir situaciones personales con sus iguales. De esta manera, las y los adolescentes refieren que han sido capaces de sentir con los otros y de mejorar la cohesión como grupo. Asimismo, cabe destacar que además de las competencias 
Desarrollo de competencias socioemocionales mediante plataforma tecnológica (Programa emoTIC): valoración de los beneficios del programa según los participantes

emocionales y las habilidades sociales, manifestaron haber mejorado su autoestima, actitud relacionada con altos niveles de bienestar subjetivo y bajos niveles de problemas emocionales y conductuales (de la Barrera et al., 2019; Escoda y Alegre, 2016).

La tercera hipótesis hacía referencia al atractivo del programa y las posibles mejoras. Respecto al atractivo del programa, se planteó que estaría relacionado con el uso de las tecnologías. Los estudios sugieren que la gamificación y el uso de tecnologías podrían resultar atractivos para los adolescentes (Wouters et al., 2013) y los resultados de este estudio parecen ir parcialmente en la línea. En este sentido, las personas participantes indicaron haberse divertido utilizando emoTIC y les gustaron aspectos relacionados con la mecánica del juego y la estética de la aplicación. Sin embargo, los aspectos más atractivos para están referidos a los contenidos tratados y a los aprendizajes obtenidos. El alumnado manifestó el atractivo de las misiones (sesiones grupales realizadas en clase) y los entrenamientos (actividades individuales realizadas en casa), así como de juegos proporcionados como refuerzo tras la realización de las actividades. Asimismo, consideraron que la incorporación de las competencias emocionales les había resultado atractiva y útil para su vida. En este sentido, las valoraciones de los y las adolescentes parecen reforzar la importancia del fomento de las competencias socioemocionales en el contexto escolar (Fomina et al., 2020; Villegas y Raffae1li, 2018). Respecto a las posibles mejoras del programa, los resultados van en la línea de la hipótesis planteada y sugieren que podría mejorarse la mecánica, el diseño y el funcionamiento del programa. Proponen mejorar la mecánica de juego incorporando un mejor sistema de distribución de puntos y evitando que sea repetitivo. Respecto al diseño, sugieren mejorar los gráficos, incluir música, añadir avatares, cambiar algunos personajes e incorporar otro tipo de ropa; es decir, proponen que el juego sea más inmersivo y lúdico. En relación al funcionamiento, recomiendan la eliminación de códigos para el avance del juego, lograr que los audios de la aplicación funcionen bien y que se pueda guardar la partida. Esto es, cuestiones técnicas para mejorar el funcionamiento de la propia aplicación.

Las valoraciones proporcionadas por los y las adolescentes participantes en relación a emoTIC han sido utilizadas para desarrollar una nueva versión, emoTIC SPACE. Principalmente, se han realizado cambios en dos bloques: (1) aspectos técnicos y (2) contenidos. En relación al primer bloque, se han corregido los errores técnicos, se ha mejorado el diseño gráfico del juego y se ha transformado en una herramienta autoaplicable accesible desde cualquier dispositivo tecnológico (p. ej., ordenadores, teléfonos inteligentes, pizarras electrónicas o tabletas). En relación al segundo bloque, se han incorporado más áreas de trabajo, por ejemplo, la toma de perspectiva, el aprecio a la diversidad, el manejo del estrés, la percepción de autoeficacia y la asertividad; y se proporciona más feedback en todas las actividades que realizan los participantes. Por tanto, la nueva versión de emoTIC es un serious game interactivo, con diversos retos y actividades con un diseño gráfico atractivo que, además, proporciona información de acuerdo al desempeño. Se ha diseñado de forma que sea lo más inmersivo posible para lograr una mayor implicación y motivación. Así, se realizan todas las actividades en el marco de un entorno ficticio que se desarrolla en cuatro Territorios, cada uno con un paisaje distinto, en el planeta E-MOOD. Un hilo argumental acompaña a través de junglas sombrías y montañas nevadas que harán que se sumerja en la historia y experimenten este programa como si fuera un estimulante videojuego. Además, el propio emoTIC SPACE tiene incorporado un sistema de refuerzo, premiando sus participantes cuando completan un reto correctamente.

Este estudio no está exento de limitaciones. Primero, únicamente se contempla la percepción subjetiva que realizan las personas participantes sobre sus aprendizajes. Sería necesario utilizar otro tipo de medidas de forma conjunta, como los autoinformes o las tareas de ejecución, que determinen la mejora en el desarrollo de las competencias, además de las propias valoraciones. En segundo lugar, sería necesario realizar una valoración del programa a largo plazo para analizar si los aprendizajes adquiridos se han integrado y permanecen en el tiempo. A pesar de las limitaciones, este estudio presenta tres fortalezas. En primer lugar, se ofrece un programa de educación socioemocional para adolescentes que incluye la tecnología para su implementación, aumentando así su atractivo. En segundo lugar, se incorpora la percepción de las personas participantes acerca de los aprendizajes obtenidos. Finalmente, se incluye un análisis de los aspectos más atractivos del programa y de las posibles mejoras que han sido incluidas en una nueva versión de emoTIC.

En conclusión, el programa de educación socioemocional mediante plataforma tecnológica llamado emoTIC se muestra útil para mejorar las competencias emocionales, las habilidades sociales y el crecimiento personal en la adolescencia. Al incorporar la tecnología en su implementación, aumenta el atractivo que tiene en esta etapa evolutiva y mejora su compromiso con el programa. Por todo ello, se considera un programa idóneo para ser llevado a cabo en el contexto educativo.

\section{Conclusión}

Para finalizar, resaltar una serie de conclusiones sobre la utilidad del programa emoTIC que se derivan del presente estudio. En primer lugar, se ofrece un programa para el desarrollo de las competencias socioemocionales que se 
realiza sobre una plataforma tecnológica. La inclusión de la tecnología y la gamificación en el área de las competencias socioemocionales conlleva un gran avance para la intervención y la promoción de la salud. En este sentido, permiten hacer más atractivos los programas de educación emocional en la adolescencia y aumentan su adherencia (Almeida, 2020; Iaosanurak et al., 2016). El presente estudio contribuye a evidenciar el impacto positivo que tienen las tecnologías para el desarrollo de las competencias socioemocionales en la adolescencia. En segundo lugar, se han analizado las valoraciones subjetivas de los y las adolescentes en relación al programa emoTIC, lo que permite explorar los aprendizajes adquiridos desde su propia perspectiva. Tras el estudio, se aporta mayor evidencia sobre la utilidad de la información que nos proporcionan las personas que participan, dado que permiten enriquecer los programas socioemocionales y guiar los cambios que se han de realizar para mejorarlos (Postigo-Zegarra, et al., 2019; Twining et al., 2017). Finalmente, el programa emoTIC atiende la necesidad del desarrollo socioemocional de los y las adolescentes, aspecto que se ha considerado tan esencial como el académico en el contexto escolar (Fomina et al., 2020). Las competencias emocionales, las habilidades sociales y el crecimiento personal son factores protectores de la salud mental adolescente y su desarrollo mediante programas atractivos implementados a través de las tecnologías pueden prevenir la aparición de problemas psicológicos en la adolescencia. Tras este estudio, se ofrece un programa socioemocional que permite promover la salud mental adolescente y favorecer las relaciones con los iguales en el contexto escolar. De esta manera, las implicaciones del programa van más allá del alumnado y pueden repercutir positivamente en otros agentes del contexto escolar, como son el profesorado, y en el seno familiar de los y las adolescentes.

\section{Agradecimientos}

Agradecemos a las familias, adolescentes y docentes su participación, su colaboración y el apoyo prestado.

\section{Financiación}

Esta investigación ha sido realizada gracias al apoyo del Ministerio de Ciencia, Innovación y Universidades (PSI2017-84005-R), por la Agencia Estatal de Investigación -AEI- y por la Unión Europea a través del Fondo Europeo de Desarrollo Regional -FEDER; así como la beca predoctoral de investigación otorgada por la Generalitat Valenciana y el Fondo Social Europeo (ACIF/2018/033).

\section{Referencias}

Almeida, F. (2020). Adoption of a serious game in the developing of emotional intelligence skills. Europe- an Journal of Investigation in Health, Psychology and Education, 10(1), 30-43. https:// doi.org/10.3390/ejihpe10010004

Baldwin, S.A. y Hoffmann, J.P. (2002). The dynamics of self- esteem: A growth curve analysis. Journal of Youth and Adolescence, 31(2), 101-113. https:// doi.org/https://doi.org/10.1023/A:1014065825598

Boyle, E.A., Hainey, T., Connolly, T.M., Gray, G., Earp, J., Ott, M., Lim, T., Ninaus, M., Ribeiro, C. y Pereira, J. (2016). An update to the systematic literature review of empirical evidence of the impacts and outcomes of computer games and serious games. Computers and Education, 94, 178-192. https:// doi.org/10.1016/j.compedu.2015.11.003

Buckley, M., Storino, M. y Saarni, C. (2003). Promoting emotional competence in children and adolescents: Implications for school psychologists. School Psychology Quarterly, 18(2), 177-191. http://doi.org/10.1521/scpq.18.2.177.21855

Cejudo, J., López-Delgado, M.L. y Losada, L. (2019). Effectiveness of the videogame "Spock" for the improvement of the emotional intelligence on psychosocial adjustment in adolescents. Computers in Human Behavior, 101, 380-386. https:// doi.org/10.1016/j.chb.2018.09.028

De la Barrera, U., Schoeps, K., Gil-Gómez, J.A. y Montoya -Castilla, I. (2019). Predicting adolescent adjustment and well-being: The interplay between socioemotional and personal factors. International Journal of Environmental Research and Public Health, 16, 4650. https://doi.org/10.3390/ ijerph16234650

De Wied, M., Branje, S. y Meeus, W. (2007). Empathy and conflict resolution in friendship relations among adolescents. Aggressive Behavior, 33, 48-55. https://doi.org/10.1002/ab.20166

Denzin, N.K. y Lincoln, Y.S. (2008). Collecting and Interpreting Qualitative Materials ( $3^{\mathrm{a}}$ ed). Sage Publications Ltd.

Elipe, P., Ortega, R., del Rey, R. y Mora-Merchán, J.A. (2011). Inteligencia emocional percibida y bullying en adolescentes. En P. FernándezBerrocal, N. Extremera, R. Palomera, D. RuizAranda, J. M. Salguero y R. Cabello (Eds.), Inteligencia Emocional: 20 Años de Investigación y Desarrollo. Fundación Marcelino Botín.

Escoda, N.P. y Alegre, A. (2016). Does emotional intelligence moderate the relationship between satisfaction in specific domains and life satisfaction? International Journal of Psychology and Psychological Therapy, 16(2), 131-140. https:// doi.org/10.6018/rie.36.1.273131

Estevez, E., Jimenez, T. y Segura, L. (2019). Emotional intelligence and empathy in aggressors and victims of school violence. Journal of Educational 
Desarrollo de competencias socioemocionales mediante plataforma tecnológica (Programa emoTIC): valoración de los beneficios del programa según los participantes

Psychology, 111, 488-496. https:// doi.org/10.1037/edu0000292

Fernández-Berrocal, P. y Extremera, N. (2015). Más Aristóteles y menos Prozac: de la inteligencia emocional a la felicidad. En P. Fernández-Berrocal, N. Extremera, R. Palomera, D. Ruiz-Aranda, J. M. Salguero y R. Cabello (Eds.), De la Neurona a la Felicidad. Diez Propuestas Desde la Inteligencia Emocional. Fundación Marcelino Botín.

Fomina, T., Burmistrova-Savenkova, A. y Morosanova, V. (2020). Self-regulation and psychological wellbeing in early adolescence: A two-wave longitudinal study. Behavioral Sciences, 10, 67. https:// doi.org/10.3390/bs10030067

Garaigordobil, M. y Maganto, C. (2011). Empatía y resolución de conflictos durante la infancia y la adolescencia. Revista Latinoamericana de Psicología, 43(2), 255-266.

Grist, R., Croker, A., Denne, M. y Stallard, P. (2019). Technology delivered interventions for depression and anxiety in children and adolescents: A systematic review and meta-analysis. Clinical Child and Family Psychology Review, 22(2), 147-171. https://doi.org/10.1007/s10567-018-0271-8

Hodzic, S., Scharfen, J., Ripoll, P., Holling, H. y Zenasni, F. (2018). How efficient are emotional intelligence trainings: A meta-analysis. Emotion Review, 10, 138-148. doi.org/10.1177/1754073917708613

https://

Iaosanurak, C., Chanchalor, S. y Murphy, E. (2016). Social and emotional learning around technology in a cross-cultural, elementary classroom. Education and Information Technologies, 21, 1639-1662. https://doi.org/10.1007/s10639-015-9406-4

Joseph, D.L. y Newman, D.A. (2010). Emotional intelligence: An integrative meta-analysis and cascading model. Journal of Applied Psychology, 95, 54 -78. doi:10.1037/a0017286

Kahn, J., Ducharme, P., Rotenberg, A. y GonzalezHeydrich, J. (2013). "RAGE-Control": A game to build emotional strength. Games for Health Journal, 2(1), 53-57. https://doi.org/10.1089/ g4h.2013.0007

Kong, F., Gong, X., Sajjad, S., Yang, K. y Zhao, J. (2019). How is emotional intelligence linked to life satisfaction? The mediating role of social support, positive affect and negative affect. Journal of Happiness Studies, 20(8), 2733-2745. https:// doi.org/10.1007/s10902-018-00069-4

Levesque, D.A., Johnson, J.L. y Prochaska, J.M. (2017). Teen choices, an online stage-based program for healthy, nonviolent relationships: Development and feasibility trial. Journal of School Violence, 16 (4), 376-385. $\quad$ https:// doi.org/10.1080/15388220.2016.1147964

Mayer, J.D. y Salovey, P. (1997). What is Emotional Intelligence? En P. Salovey y D. Sluyter (Eds.), Emotional Development and Emotional Intelligence: Implications for Educators (pp. 3-31). Basic Books.

Mayer, J.D., Caruso, D.R. y Salovey, P. (2016). The ability model of emotional intelligence: Principles and updates. Emotion Review, 8(4), 290-300. https:// doi.org/10.1177/1754073916639667
Mayer, J.D., Salovey, P. y Caruso, D.R. (2000). Models of Emotional Intelligence. En R.J. Sternberg (Ed.), Handbook of Intelligence (pp. 396-420). Cambridge University Press.

Mayer, J.D., Salovey, P. y Caruso, D.R. (2004). Emotional intelligence: Theory, findings, and implications. Psychological Inquiry, 15, 197-215. https:// doi.org/10.1207/s15327965pli1503

Nussbaum, M. y Beserra, V.D.S. (2014). Educational videogame design. Proceedings - IEEE 14th International Conference on Advanced Learning Technologies, ICALT 2014, 2-3. https://doi.org/10.1109/ ICALT.2014.9

Oliva-Delgado, A. (2011). Apego en la adolescencia. Acción Psicológica, 8(2), 55-65.

Peachey, A.A., Wenos, J. y Baller, S. (2017). Trait emotional intelligence related to bullying in elementary school children and to victimization in boys. OTJR Occupation, Participation and Health, 37 (4), 178-187. https:// doi.org/10.1177/1539449217715859

Postigo-Zegarra, S., Schoeps, K., Montoya-Castilla, I. y Escartí, A. (2019) Emotional education program for adolescents (PREDEMA): Evaluation from the perspective of students and effects on socioaffective competences. Infancia y Aprendizaje, 42(2), 303-336. doi.org/10.1080/02103702.2019.1578925

Rosenberg, M. (1965). Society and The Adolescent Selfimage. Princeton University Press.

Saarni, C. (2000). Emotional competence: A developmental perspective. En R. Bar-On y J.D.A. Parker (Eds.), The Handbook of Emotional Intelligence: Theory, Development, Assessment, and Application at Home, School, and in the Workplace (pp. 68-91). Jossey-Bass.

Sánchez-Álvarez, N., Extremera, N. y Fernández-Berrocal, P. (2016). The relation between emotional intelligence and subjective well-being: A meta- analytic investigation. Journal of Positive Psychology, 11, $276-285$. $10.1080 / 17439760.2015 .1058968$

Sarrionandia, A. (2017). Effects of an emotional intelligence program on socioemotional factors and psychosomatic symptoms. Revista Latinoamericana de Psicología, 49, 110-118. https:// doi.org/10.1016/j.rlp.2015.12.001

Schoeps, K., Villanueva, L., Prado-Gascó, V.J. y MontoyaCastilla, I. (2018). Development of emotional skills in adolescents to prevent cyberbullying and improve subjective well-being. Frontiers in Psychology, 9, 2050. https://doi.org/10.3389/ fpsyg.2018.02050

Smith, E.R., Mackie, D.M. y Claypool, H. (2014). Social Psychology. Psychology Press.

Twining, P., Heller, R.S., Nussbaum, M. y Tsai, C.C. (2017). Some guidance on conducting and reporting qualitative studies. Computers \& Education, 106, A1-A9. https://doi.org/10.1016/ j.compedu.2016.12.002

Viguer, P., Cantero, M.J. y Bañuls, R. (2017). Enhancing emotional intelligence at school: Evaluation of the effectiveness of a two-year intervention program 
in Spanish pre-adolescents. Personality and Individual Differences, 113, 193-200. https:// doi.org/10.1016/j.paid.2017.03.036

Villegas, E. y Raffaelli, M. (2018). Experiencing and learning about emotions: A longitudinal analysis of youth program participants. Journal of Youth and Adolescence, 47(8), 1684-1696. https:// doi.org/10.1007/s10964-018-0885-7

World Medical Association. (2013). World Medical Association Declaration of Helsinki: Ethical principles for medical research involving human subjects. Journal of American Medical Association, 310, 2191-2194.

Wouters, P., van Nimwegen, C., van Oostendorp, H. y van Der Spek, E.D. (2013). A meta-analysis of the cognitive and motivational effects of serious games. Journal of Educational Psychology, 105 (2), 249-265. https://doi.org/10.1037/a0031311 